\title{
Investigation of radiative coupling and of enlarged decay rates of nuclear oscillators
}

\author{
W. Potzel, * U. van Bürck, P. Schindelmann, and G. M. Kalvius \\ Physik-Department E15, Technische Universität München, D-85748 Garching, Germany \\ G. V. Smirnov \\ Russian Research Center “Kurchatov Institute," 123182 Moscow, Russia \\ E. Gerdau, Yu. V. Shvyd'ko, and H. D. Rüter \\ II. Institut für Experimentalphysik, Universität Hamburg, D-22761 Hamburg, Germany \\ O. Leupold \\ II. Institut für Experimentalphysik, Universität Hamburg, D-22761 Hamburg, Germany \\ and European Synchrotron Radiation Facility (ESRF), POB 220, F-38043 Grenoble, France
}

(Received 6 November 2000; published 14 March 2001)

\begin{abstract}
The nuclear forward scattering of synchrotron radiation (SR) of two spatially separated stainless-steel foils has been investigated. Immediately after the SR pulse a nuclear exciton extending over both targets is formed, which then decays under the influence of radiative coupling of the nuclear oscillators both within and between the targets. Cooling one target to low temperatures causes the Mössbauer linewidth to increase by inhomogeneous broadening, which leads to a rapid dephasing of the nuclear oscillators of this system. In general, the influence of the radiation field of the upstream oscillators on the oscillators of the downstream target causes the latter to radiate at a high decay rate (steep slope) at early times. Surprisingly, for the combined targets, the initial decay can be less steep than that of the inhomogeneously broadened sample alone if this broadening is sufficiently large. In addition, when the upstream oscillators are broadened in energy they may cause the downstream oscillators to emit only very little intensity at late times. In the experiments, this influence on the downstream sample was changed by varying the inhomogeneous resonance broadening and the resonance frequencies of the targets. Drastic changes of the time evolution of the nuclear forward scattering were observed, which are discussed within the dynamical theory.
\end{abstract}

DOI: 10.1103/PhysRevA.63.043810

PACS number(s): 42.50.Fx, 42.25.Bs, 42.25.Hz, 76.80.+y

\section{INTRODUCTION}

Coupled oscillators play an essential role in many different areas of physics. They are important in classical dynamics [1], microwaves [2], and lasers [3,4], and probably already had a large impact at very early times of the Universe $[5,6]$. The application of the Mössbauer effect [7-11] and, in particular, more recently the availability of strong synchrotron radiation (SR) sources made it possible to study nuclear oscillators that are coupled by $\gamma$ radiation transmitted through resonant absorbers [12-16]. Mössbauer transitions are in many respects ideal oscillators. For example, they exhibit strong scattering power, corresponding in the case of the $14.4-\mathrm{keV}$ resonance in ${ }^{57} \mathrm{Fe}$ to that of several hundreds of electrons, and they are well isolated in energy. The relatively long lifetimes of Mössbauer nuclear levels in the nanosecond range make it possible to excite the Mössbauer transitions by short and highly brilliant pulses of SR. The propagation of the SR pulse through a system of resonant oscillators $[17,18]$ can be studied by the time evolution of coherent delayed radiation, which is reemitted practically background-free in a nuclear forward scattering experiment $[19,12-14]$.

Even though modern synchrotron facilities provide very intensive x-ray beams, in most cases of nuclear forward scat-

\footnotetext{
*Email address: wpotzel@ph.tum.de
}

tering of synchrotron radiation (NFSSR) experiments the targets have to be thick in order to obtain a reasonable NFS signal. Typically the Mössbauer thickness $T=n \sigma_{0} f$ is $\geqslant 10$, where $n$ is the number of resonant nuclei per unit area, $\sigma_{0}$ is the maximal resonance cross section of the nuclear transition, and $f$ is the Lamb-Mössbauer factor. Such big ensembles of identical nuclear oscillators exhibit interesting properties, in particular because the individual oscillators may be coupled via the radiation field. Although the targets have large $T$ values, each prompt SR pulse typically creates only one (and more often no) resonant excitation in such a target. One might conclude that this excitation is localized at just one nucleus which has been lifted into the excited state. This, however, is not the case in coherent forward scattering, where it is impossible to identify the scattering nucleus. Rather, for each individual nucleus in the spatially extended target there exists the same small probability amplitude that this nucleus is excited while all other nuclei are left in the ground state. All these small probability amplitudes are phased by the prompt SR flash and have to be added to obtain the total probability amplitude for a photon to be absorbed by the nuclei in the target. Such a collective nuclear excitation, which is phased in time by the SR flash and can extend over the whole target, is known as a nuclear exciton $[20,21]$.

Nuclear excitons exhibit interesting cooperative properties which characterize the time dependence of NFSSR. The 
intensity reemitted immediately after the SR flash (at $t=0$ ) is proportional to $T^{2}$. This might be expected on the basis of kinematical theory, which takes into account only the interaction with the primary radiation. In this case, the field amplitudes of all nuclei have to be added and the total amplitude has to be squared to obtain the intensity. However, additional aspects come into play that have to be described by a dynamical theory which takes into account the interaction of secondary radiation with the nuclei. The dynamical effects already manifest themselves at early times $(t \rightarrow 0)$ : the initial decay rate is enhanced leading to a logarithmic slope of the intensity $I(t), d \ln I(t) /\left.d t\right|_{t=0}$, which is more negative than $-1 / \tau$, where $\tau$ is the lifetime of the excited nuclear state of an individual nucleus. This effect is known as coherent enhancement or speed-up of the initial decay [20,21]. Further, dynamical beats (DB's) develop in the time dependence of the reemitted radiation intensity. They are an important feature of NFSSR. Both initial speed-up and DB's are due to coupling of the individual oscillators in the target via the propagating radiation field $[17,18,13,20-26]$. A quantitative description for the case of SR pulses was first derived in Ref. [20].

The initial decay rate of the NFSSR signal can also be influenced by quite a different process: inhomogeneous broadening. It causes dephasing of the resonant oscillators. Therefore the coherence within the nuclear exciton is partially destroyed and the coherent signal is reduced. The result of combinations of coherent enhancement and dephasing we call enlarged decay rates.

In the present paper we describe a comprehensive study of radiative coupling and of enlarged decay rates of big ensembles of nuclear oscillators. We will focus on two ensembles represented by two spatially separated targets $A$ and $B$ mounted downstream behind each other in a monochromatized SR beam. The time dependence of NFSSR by the composite target $(A+B)$ is measured [27]. Two types of radiative coupling can be distinguished in such a system, although this distinction is somewhat superficial: coupling within each individual target and coupling between targets $A$ and $B$. Coupling between the different nuclei within each target occurs because the delayed radiation reemitted from the upstream part of the target interacts with the parts further downstream. Coupling between targets $A$ and $B$ occurs because the nuclei in the downstream target $B$ can resonantly interact with the delayed radiation reemitted from the upstream target $A$. Thus target $B$ is excited by the SR flash and, in addition, it is driven by the delayed electromagnetic field reemitted from target $A$. However, it should be emphasized that radiative coupling in NFSSR via the propagating radiation field is a one-way street: in both types of coupling, upstream nuclei can influence downstream nuclei, but not vice versa.

Of highest interest is the experimental possibility of changing the coupling between the targets $A$ and $B$, which for brevity we will call $A B$ coupling. In our scattering system the changes in $A B$ coupling will be accomplished in two different ways: (a) inhomogeneous broadening will be increased in one target $(A)$ by changing the hyperfine interaction at its resonant nuclei when lowering the temperature of target $A$; (b) a shift between the resonance frequencies of the

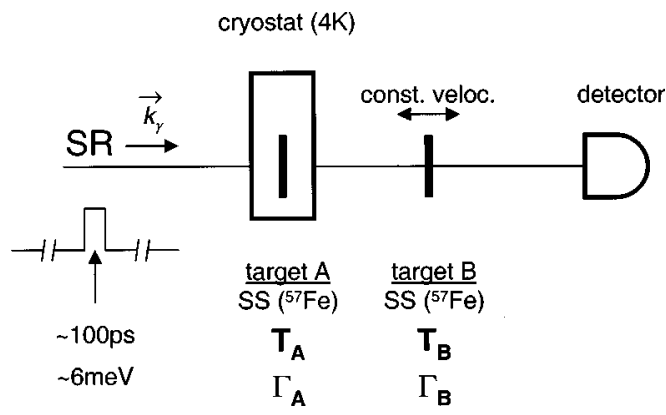

FIG. 1. Experimental setup for investigating the time response of the ${ }^{57} \mathrm{Fe}$ nuclei in two separated SS targets $A$ and $B$ to the excitation by a synchrotron radiation pulse. Target $A$ (Mössbauer thickness $T_{A}$, linewidth $\Gamma_{A}$ ) can be cooled to liquid-He temperatures in a cryostat. Target $B$ (Mössbauer thickness $T_{B}$, linewidth $\Gamma_{B}$ ) can be moved at constant velocity by a double-loudspeaker drive.

nuclei in both targets will be applied by moving one target $(B)$ at a constant Doppler velocity.

In a combined target system, the reemitted intensity $I(t)$ is always increased at very early times $(t \rightarrow 0)$ due to the greater Mössbauer thickness of the nuclear ensemble as compared to each individual target alone. This increased intensity originates from constructive interference. Usually, as, for example, in the case of resonances with nearly natural linewidth, the $A B$ coupling causes an enhancement of the initial decay rate, i.e., the logarithmic slope $d \ln I /\left.d t\right|_{t=0}$ of the intensity $I$ at $t \rightarrow 0$ is steeper than that of the time dependence for each individual target. In addition, in this case considerable intensity persists until late times $t \gg \tau$, although modulated by the DB's. In contrast, it will turn out that when target $A$ is inhomogeneously broadened the initial slope may become even less steep than that of the broadened target $A$ alone if the inhomogeneous broadening of target $A$ is sufficiently large. Furthermore, rather little intensity may be observed at late times of the NFSSR pattern also for the combined target system $(A+B)$.

In addition, the question will be discussed whether the sequence of targets is of importance. It will be shown that the results of the present experiments are independent of the target sequence. Future measurements are indicated where this will no longer be the case. All experimental results are quantitatively described by the dynamical theory of nuclear forward scattering.

\section{EXPERIMENTAL DETAILS}

Targets $A$ and $B$ were made from stainless-steel (SS) foils of composition $\mathrm{Fe}_{55} \mathrm{Cr}_{25} \mathrm{Ni}_{20}$ enriched to $95 \%$ in ${ }^{57} \mathrm{Fe}$. The thickness of the foils was $-2.5(A)$ and $\sim 1.3 \mu \mathrm{m}(B)$. The SR experiments were performed at HASYLAB (Hamburg) at the wiggler beamline BW4. In the five-bunch mode of the storage ring, the time window between the SR bunches was $\sim 190$ ns. The experimental setup is depicted in Fig. 1. After monochromatization of the SR of $14.4 \mathrm{keV}$ to a bandwidth of $6 \mathrm{meV}$ by a four-bounce high-resolution monochromator $[28,29]$, the SR pulse of typically several $100 \mathrm{ps}$ in length excites the nuclei in target $A$ of Mössbauer thickness $T_{A}$ and 


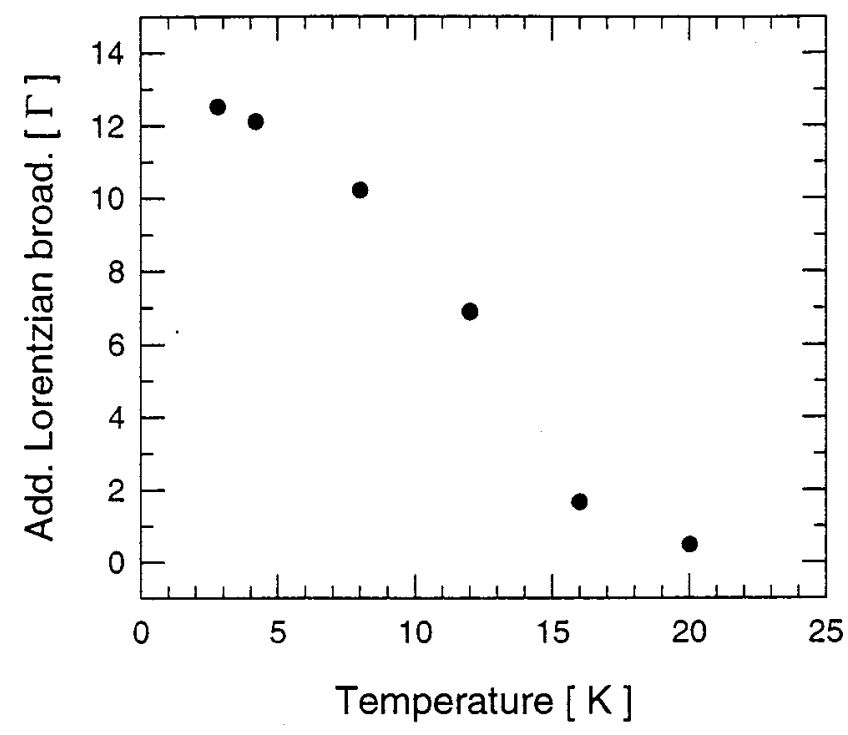

FIG. 2. Increase of the additional Lorentzian broadening $\Delta_{A}$ of the nuclear resonance in target $A$ when the sample temperature is lowered from 20 to $2.8 \mathrm{~K}$. The results for $\Delta_{A}$ were obtained from computer fits to the NFSSR data shown in Fig. 4 below with Lorentzian broadening alone being used as a model to describe the actual line broadening observed.

linewidth $\Gamma_{A}$. This target is mounted in a liquid-He cryostat and may be cooled to cryogenic temperatures. Thereafter the SR pulse hits target $B$ with thickness $T_{B}$ and linewidth $\Gamma_{B}$. This target is kept at room temperature and may be moved at constant velocities in the range of $0-10 \mathrm{~mm} / \mathrm{s}$ by a Mössbauer double-loudspeaker drive. A fast-avalanche photodiode [30-32] is used to detect the radiation transmitted by both targets in the forward direction. The time dependence of this nuclear forward scattering intensity is digitally stored.

The SS material used for the targets $A$ and $B$ is known as a single-line absorber in Mössbauer spectroscopy with a slightly broadened resonance linewidth of $\sim 1.5 \Gamma$ at temperatures between room temperature and $\sim 20 \mathrm{~K}$. The natural linewidth of $\Gamma=0.098 \mathrm{~mm} / \mathrm{s}$ corresponds to about $5 \mathrm{neV}$. The linewidth rapidly increases when the temperature is lowered below $20 \mathrm{~K}$. In first approximation the NFSSR time dependence of the SS sample at lower temperatures (target A) can be fitted when increasing the effective linewidth by an additional Lorentzian broadening $\Delta_{A}$. As shown in Fig. 2, $\Delta_{A}$ amounts to $\sim 0.5 \Gamma$ at $20 \mathrm{~K}$ and increases to $\sim 12.5 \Gamma$ when the temperature reaches $\sim 3 \mathrm{~K}$. This rapid rise has been observed in Mössbauer spectroscopy as well [33-35]. It is caused by a change in hyperfine interactions, most probably by electronic relaxation effects [35]. In this article we will not investigate this temperature dependence. We just use this behavior as a convenient way to change the linewidth of target $A$. To achieve more detailed fits, in addition to the dominating temperature-dependent Lorentzian broadening, a small hyperfine splitting by a random magnetic field was introduced in the fitting procedure. From room temperature (RT) to $20 \mathrm{~K}$ a magnetic field of $0.59 \mathrm{~T}$ was assumed. With temperatures decreasing below $20 \mathrm{~K}$, for consistent fits to all data sets recorded at the various temperatures, increasing field values had to be used, which reached $1.84 \mathrm{~T}$ at $2.8 \mathrm{~K}$.

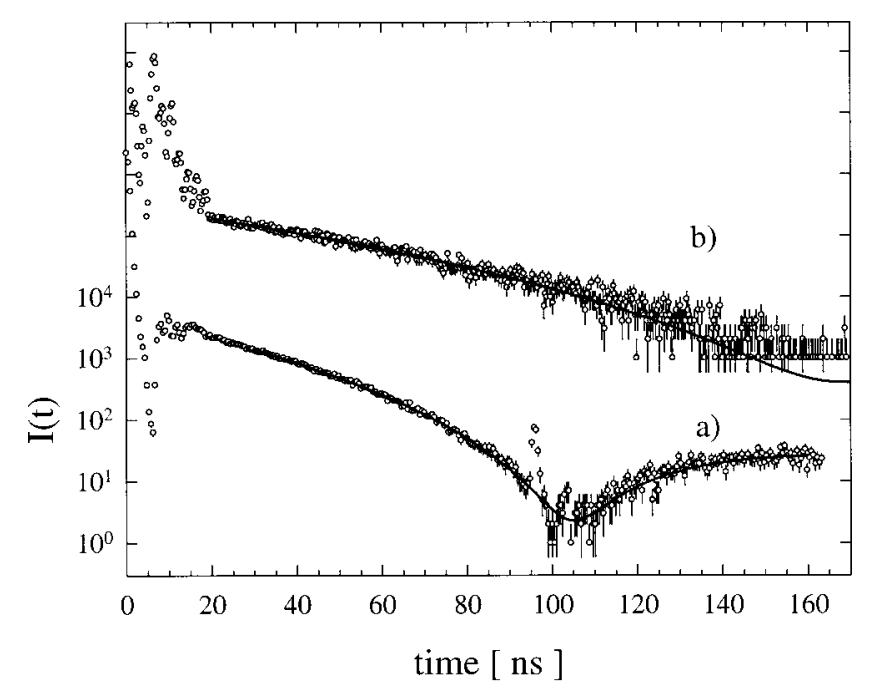

FIG. 3. Comparison of the NFSSR of target $A$ (a) and of target $B$ (b) both at room temperature. The thicknesses were $\sim 2.5 \mu \mathrm{m}$ $\left(T_{A} \approx 17.9\right)$ for target $A$ and $\sim 1.3 \mu \mathrm{m}\left(T_{B} \approx 9.3\right)$ for target $B$. The small spike at $\sim 95 \mathrm{~ns}$ is due to a spurious bunch. In the time window up to $\sim 20 \mathrm{~ns}$ counting is spoiled by electronic gating effects and spurious bunches. The data sets are artificially shifted for a better display.

The change of the Lamb-Mössbauer factor between room temperature and $2.8 \mathrm{~K}$ was taken into account in the fits. All time dependences were fitted by the NFS theory implemented in the computer code MOTIF [15].

\section{RESULTS AND DISCUSSION}

\section{A. NFSSR of SS targets with almost natural linewidth}

Figure 3 shows the NFSSR patterns of each of the SS foils at room temperature. A foil of $\sim 2.5 \mu \mathrm{m}$ thickness $\left(T_{A} \approx 17.9\right)$ was used as target $A$ (see Fig. 1$)$. The first minimum of the dynamical beats occurs at $\sim 105$ ns [see Fig. 3(a)]. The foil of target $B$ was $\sim 1.3 \mu \mathrm{m}$ thick $\left(T_{B} \approx 9.3\right)$. With this thickness the initial slope (to be seen in Fig. 3 only at $\sim 20 \mathrm{~ns}$ because of spoiled counting at times $t<20 \mathrm{~ns}$ ) is rather modest and the first minimum of the DB's occurs at $\sim 170$ ns [see Fig. 3(b)].

The delayed radiation field being reemitted by the target of Mössbauer thickness $T$ is given by [20]

$$
E(t) \propto e^{-t / 2 \tau} e^{i \omega_{0} t} A(t),
$$

with

$$
A(t)=T \sigma(T t / \tau)=T J_{1}(\sqrt{T t / \tau}) / \sqrt{T t / \tau}
$$

where $\tau$ is the lifetime of the nuclear state (for ${ }^{57} \mathrm{Fe} \tau$ $\approx 141 \mathrm{~ns}$ ), $\omega_{0}$ is the resonance frequency (for ${ }^{57} \mathrm{Fe} \omega_{0} \approx 2.1$ $\left.\times 10^{19} \mathrm{~s}^{-1}\right)$, and $J_{1}$ is the Bessel function of the first kind and first order. The $J_{1}$ modulations of $E(t)$ lead to the DB minima of the scattered intensity. If $T$ is reduced the DB minima occur at later times. 


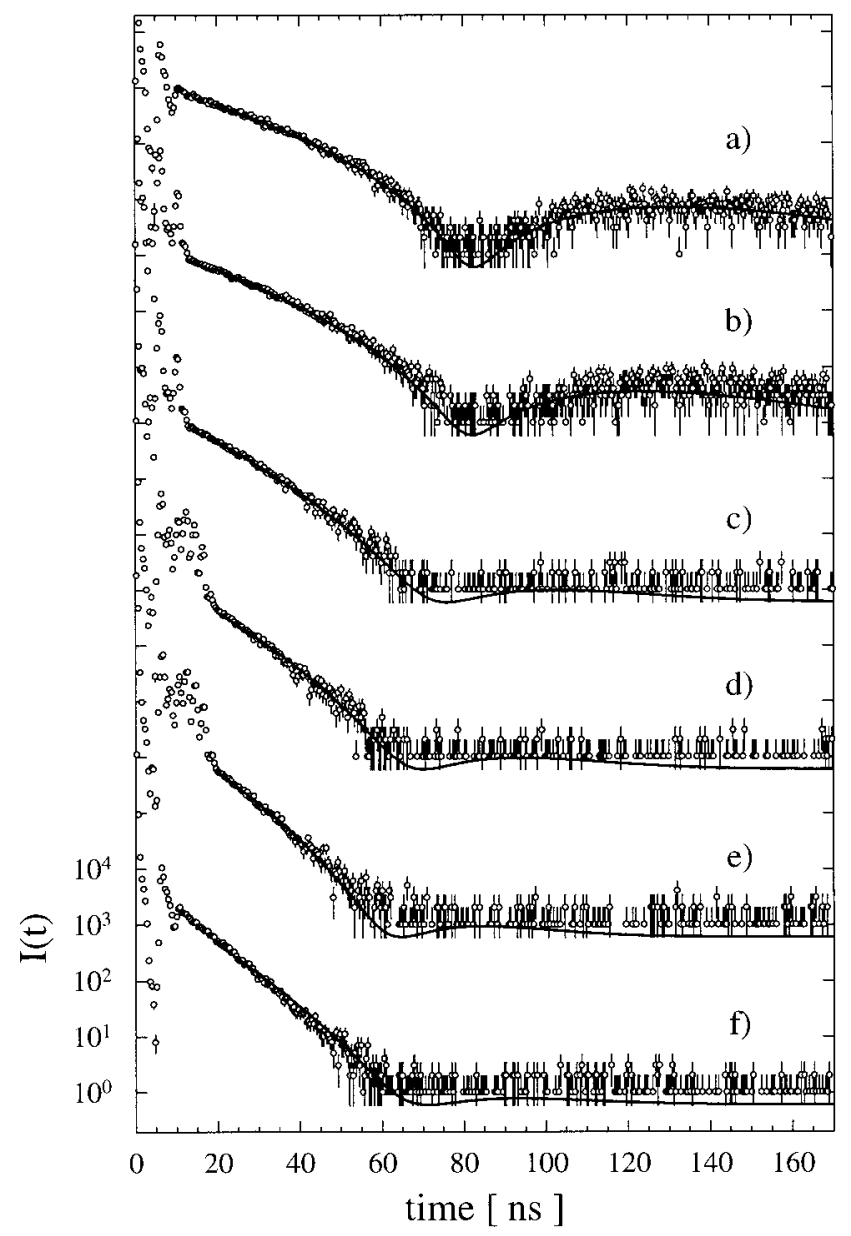

FIG. 4. NFSSR of a stainless-steel foil (target $A$ ) of $\sim 2.5 \mu \mathrm{m}$ thickness $\left(T_{A} \approx 23.6\right)$ at temperatures of 20 (a), 16 (b), 12 (c), 8 (d), 4.2 (e), and $2.8 \mathrm{~K}$ (f). Note the steep decay at low temperatures due to the rapid dephasing of the individual oscillators in the target. The data sets are artificially shifted for a better display.

For small Mössbauer thickness $T$ and/or early times, $T t / \tau \ll 1$. In this limit, or even for $T t / \tau<3$ [22], one obtains [36]

$$
E(t) \propto T e^{-(1+T / 4) t / 2 \tau} e^{i \omega_{0} t}
$$

and with $I=|E|^{2}$ the initial slope of the intensity $I$ is then given by

$$
d \ln I /\left.d t\right|_{t=0} \approx-(1 / \tau)(1+T / 4) .
$$

Equation (3) gives an enhanced scattering intensity proportional to $T^{2}$. Equation (4) describes a coherent enhancement of the initial decay rate $[20,21,25,26]$ determined by $T / 4$.

\section{B. Dephasing of nuclear oscillators in the case of inhomogeneous line broadening}

The rapid increase of the linewidth at low temperatures (see Fig. 2) causes characteristic changes of the time dependence of NFSSR. Figure 4 shows the results for target $A$ in the temperature range between 20 and $2.8 \mathrm{~K}$. At $20 \mathrm{~K}$ the first minimum of the DB's occurs at $\sim 80 \mathrm{~ns}$ in agreement with the increased thickness of $T_{A} \approx 23.6$ due to the rise of the Lamb-Mössbauer factor $f$ as compared to the RT value [see Fig. 3(a)]. When the temperature is lowered the shape of the time dependence changes markedly. The minimum of the NFSSR intensity moves to $\sim 65 \mathrm{~ns}$ and the initial slope becomes steeper. In addition, the time dependences recorded at 4.2 and $2.8 \mathrm{~K}$ show hardly any rise of the intensity after the minimum, in contrast to the $20-\mathrm{K}$ pattern. This is a most remarkable feature. The slight increase in $f$ of $\sim 2 \%$ between 20 and $2.8 \mathrm{~K}$ cannot be responsible for these changes. The rapid decrease of the NFSSR intensity is mainly due to inhomogeneous broadening of the resonance width in SS by hyperfine interactions. Such an inhomogeneous broadening is expected to cause a rapid dephasing between the individual oscillators, which results in a steep decay and, in addition, a drastic reduction of the coherent forward scattering at late times [13,37]. These effects are observed in Fig. 4 for continuously increasing line broadening.

In the case of inhomogeneous Lorentzian line broadening described by $q=1+\Delta_{A} / \Gamma$, Eqs. (3) and (4) are replaced by [22]

$$
E(t) \propto T e^{-(q+T / 4) t / 2 \tau} e^{i \omega_{0} t}
$$

and

$$
d \ln I /\left.d t\right|_{t=0} \approx-(1 / \tau)(q+T / 4)
$$

The forward reemitted intensity starts at the same level proportional to $T^{2}$ for $t \rightarrow 0$ independent of the broadening $q$. However, its initial enhanced decay rate is further increased by $q>1$ due to dephasing of the coherent forward scattering.

\section{Dependence of $A B$ coupling on oscillator line broadening}

We first discuss the NFSSR of both targets $(A+B)$ together, for the case that the resonance energies of $A$ and $B$ coincide. Target $B$ exhibits almost natural linewidth and will experience the radiation reemitted from target $A$, whose oscillators exhibit additional line broadening.

Figure 5 displays the time dependence of both targets $(A+B)$ together, with target $A$ at various temperatures. Target $B$ is kept at room temperature. The resonance energies of both targets were kept the same within $\leqslant 0.3 \Gamma$ by moving $\operatorname{target} B$ on a Mössbauer drive to compensate for the secondorder Doppler (SOD) shift. From our computer fits we conclude that the time dependences recorded at 20, 16, and $12 \mathrm{~K}$ [see Figs. 5(a)-5(c)] show higher initial intensities and enlarged decay rates (steeper slopes) at early times as compared to those of target $A$ alone [compare Figs. 4(a)-4(c)]. The DB minimum has shifted from $\sim 80$ to $\sim 60 \mathrm{~ns}$. With $\operatorname{target} A$ at $20 \mathrm{~K}$ [see Fig. 5(a)] the additional Lorentzian broadening is so small that the two targets together essentially act at all times as one target with total thickness $T_{A+B}=T_{A}+T_{B} \approx 32.9$. This increase in Mössbauer thickness explains the increase of initial intensity, the steeper slope, and the shift of the DB minimum. However, the time dependences obtained at 4.2 and $2.8 \mathrm{~K}$ [see Figs. 5(e) and 5(f)] look very surprising. In particular, the time pattern of the NFSSR obtained for $A+B$ together, with $A$ at $2.8 \mathrm{~K}$ [Fig. 


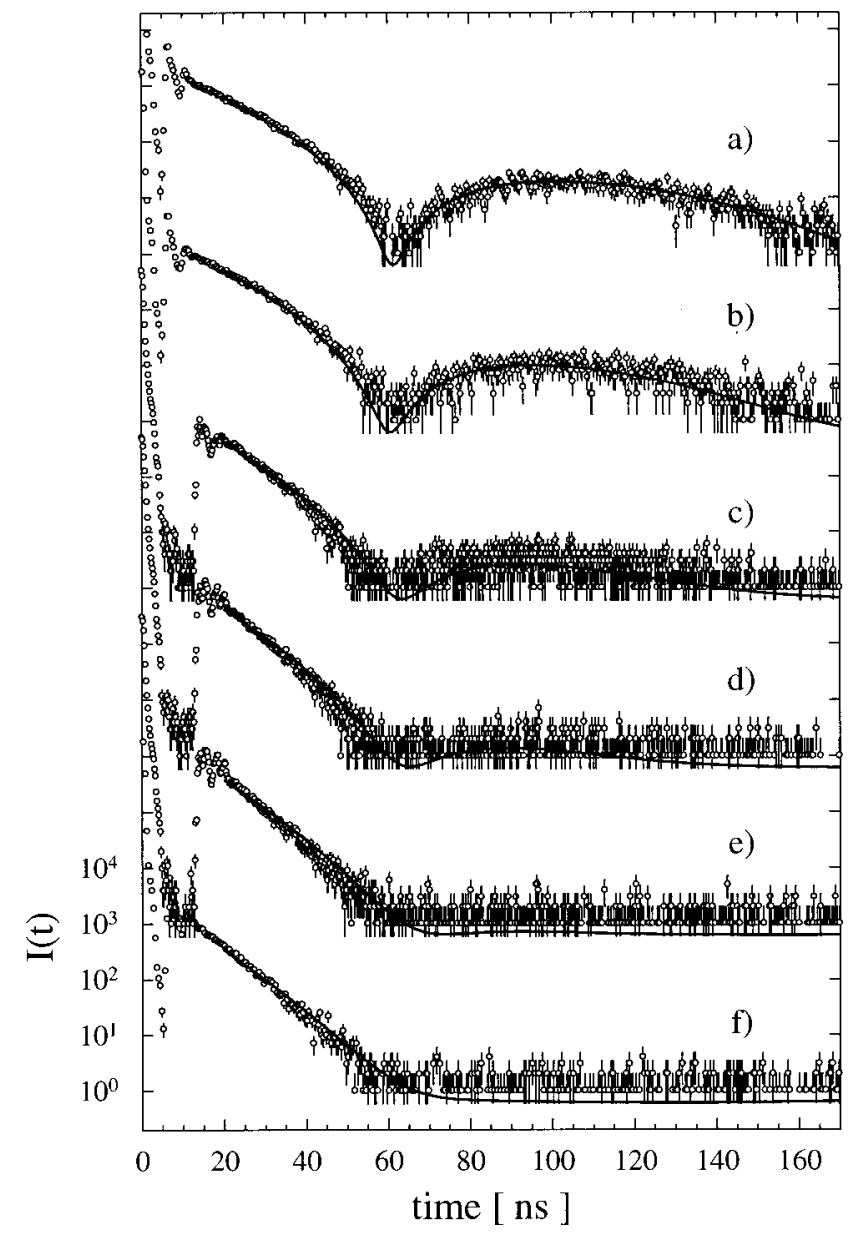

FIG. 5. NFSSR of the combined target $(A+B)$ with target $A$ at 20 (a), 16 (b), 12.5 (c), 8.4 (d), 4.2 (e), and $2.8 \mathrm{~K}$ (f). Target $B$ was kept at room temperature. The resonance energies of both targets are kept the same within $\leqslant 0.3 \Gamma$ by moving target $B$ by a Doppler drive to compensate for the SOD shift. The bottom curve looks highly similar to that of target $A$ alone in Fig. 4(f). Other details for target $A$ and target $B$ as in Figs. 4 and 3, respectively.

5(f)] looks very similar to that for $A$ alone at $2.8 \mathrm{~K}$ [see Fig. 4(f)]. In comparison with Figs. 3 and 4(f) two features appear most striking [16,38]: (1) The NFSSR of both targets $(A$ $+B)$ together shows very little intensity for times $t \geqslant 70 \mathrm{~ns}$.

(2) The initial slope of $(A+B)$ is not steeper and is maybe even slightly less steep than that of $A$ alone. These two features will be analyzed and discussed in the following.

\section{Reduction of NFSSR intensity at late times}

One might expect that, after the rapid decay of the NFSSR intensity of target $A$, the signal of target $B$ should still be strong and be characterized largely by that of a $1.3-\mu \mathrm{m}-$ thick foil [see Fig. 3(b)]. This is not the case. As Figs. 5(f) and 5(e) clearly demonstrate the NFSSR intensity behind both foils is strongly reduced at times $t \geqslant 70 \mathrm{~ns}$. Apparently, in spite of the rapid decay of target $A, A B$ coupling causes target $B$ to radiate at a high decay rate at early times $(<60$ ns) and to emit only very little intensity at later times.

These results can be understood on the basis of the equations describing the radiation field reemitted by the com- bined target $(A+B)$. The wave field that is present behind both targets $A$ and $B$ and the intensity of which is recorded in the detector is given by $[22,39]$

$$
\begin{aligned}
E(t)_{A+B} \propto & \left\{\delta(t)-(1 / 2 \tau) e^{i \omega_{B} t-\left(q_{B} / 2 \tau\right) t}\right. \\
& \times\left[T_{A} \sigma\left(T_{A} t / \tau\right) e^{i\left(\omega_{A}-\omega_{B}\right) t-\left[\left(q_{A}-q_{B}\right) 2 \tau\right] t}\right. \\
& +T_{B} \sigma\left(T_{B} t / \tau\right)-(1 / 2 \tau) T_{A} T_{B} \\
& \times \int_{0}^{t} d t^{\prime} e^{i\left(\omega_{A}-\omega_{B}\right) t^{\prime}-\left[\left(q_{A}-q_{B}\right) / 2 \tau\right] t^{\prime}} \sigma\left(T_{A} t^{\prime} / \tau\right) \\
& \left.\left.\times \sigma\left(T_{B}\left(t-t^{\prime}\right) / \tau\right)\right]\right\} .
\end{aligned}
$$

Inhomogeneous broadening of Lorentzian type is taken into account by $q_{A}$ and $q_{B}$ with $q_{A, B}=1+\Delta_{A, B} / \Gamma$. The resonance frequencies of the nuclei in the targets $A$ and $B$ are denoted by $\omega_{A}$ and $\omega_{B}$, respectively. If both targets contain nuclei with the same resonance frequency, $\omega_{A}=\omega_{B}$. Equation (7) describes four different optical paths [22,39].

(a) SR is transmitted without interaction at all. This radiation is described by the delta function $\delta(t)$.

(b) Nuclear scattering of the SR pulse is by target $A$ only, represented by the first term in the square brackets.

(c) Nuclear scattering of the SR pulse is by target $B$ only, represented by the second term in the square brackets.

(d) Nuclear scattering of the SR pulse is by $\operatorname{target} A$ and target $B$, described by the integral term in the square brackets. Radiation having first excited the nuclei and dwelt in target $A$ up to time $t^{\prime}$ excites the nuclei in the downstream target $B$ at time $t^{\prime}$. They are deexcited at time $t$. This term describes the $A B$ coupling, i.e., the influence of the radiation field of the upstream target on the downstream target. Since the integration is performed up to time $t$, this term is important also at late times even though the field of $A$ might have vanished long before time $t$. It is interesting to note that this term has an opposite sign with respect to the first two, i.e., the wave associated with it is in antiphase to the two other waves. The shift $\pi$ in the phase arises from the additional resonance scattering in target $B$ : resonance scattering by a nucleus gives $\pi / 2$ and another $\pi / 2$ is due to the coherent summation of the wavelets scattered by nuclei lying in the plane wave front of the radiation (Fresnel construction).

It is also helpful to take an alternative view of the scattering event where the optical paths (c) and (d) represented by the second and the integral term in the square brackets of Eq. (7) are combined in one path. In this view Eq. (7) describes three different radiation fields [40].

(a) SR transmitted without interaction.

(b) The radiation field amplitude reemitted by the upstream target $A$, which we denote by $A^{u}(t)$.

(c) The radiation field amplitude reemitted by the downstream target $B$, which we denote by $A^{d}(t)$. This field reflects both that target $B$ is charged by the SR pulse and that 


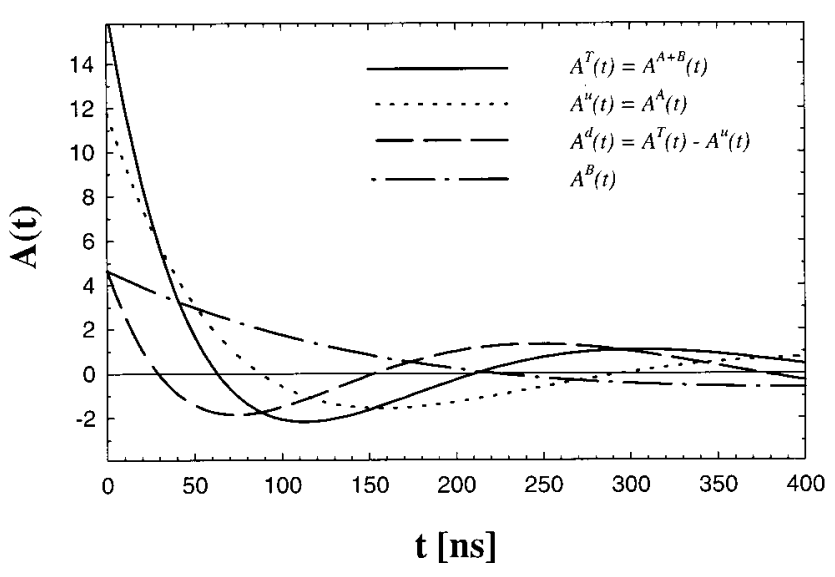

FIG. 6. Envelopes of the radiation fields according to Eq. (2) of the combined $(A+B)$ target $A^{T}(t)$, of the upstream target $A^{u}(t)$, and of the downstream target $A^{d}(t)$ for the case that $A$ is upstream of $B . A^{B}(t)$ denotes the response of target $B$ if it were present alone. Target $A, T_{A} \approx 23.6$; target $B, T_{B} \approx 9.3$. For both targets natural linewidth was assumed.

it is driven by the radiation field reemitted by $\operatorname{target} A$, i.e., it gives the total response of target $B$.

This notion will also be of importance when we discuss implications of the sequence of targets in Sec. V below.

First we study the role of $A B$ coupling in greater detail by computer simulations, and start by discussing the simple case that in both targets no additional broadening is present. Both targets are kept stationary. As a consequence, the $A B$ coupling is maximal and the two targets act as a combined target of total thickness $T_{A+B}=T_{A}+T_{B}$. Figure 6 shows the envelopes $A(t)$ of Eq. (2) for the case [40] that $A\left(T_{A} \approx 23.6\right)$ is upstream of $B\left(T_{B} \approx 9.3\right)$. Since the upstream target influences the downstream target and not vice versa, the envelope $A^{u}(t)$ of the upstream target is known as well as the total amplitude $A^{T}(t)$ of both targets combined. Thus $A^{d}(t)$ of the downstream target can be calculated as $A^{d}(t)=A^{T}(t)$ $-A^{u}(t)$. As Fig. 6 demonstrates, $A^{d}(t)$ is modified quite strongly as compared to $A^{B}(t)$, the envelope of $\operatorname{target} B$ if it were just radiating by itself without being driven by the radiation field of $A$. For example, the first zero crossing of $A^{d}(t)$ has been shifted to $\sim 30 \mathrm{~ns}$ as compared to $\sim 200 \mathrm{~ns}$ for $A^{B}(t)$. These big changes are caused by the driving action of $A$, which is described by the coupling term in Eq. (7). The radiation field $A^{d}(t)$ emitted by the downstream target $B$ results from the superposition of $A^{B}(t)$ and this coupling term.

In the more general case that target $A$ is kept at reduced temperature and exhibits additional broadening, the envelopes $A(t)$ can only be calculated numerically. The main results of preliminary simulations are the following. The radiation field of $A$ will be rapidly diminished due to dephasing of the oscillators in $A$. However, due to $A B$ coupling, target $B$ will radiate with a higher decay rate (increased slope) at early times and with little intensity at late times as compared to target $B$ alone. Thus each target will emit only weak radiation fields at late times $(t>70 \mathrm{~ns})$ : target $A$ because of rapid dephasing of its oscillators and $\operatorname{target} B$ because of $A B$

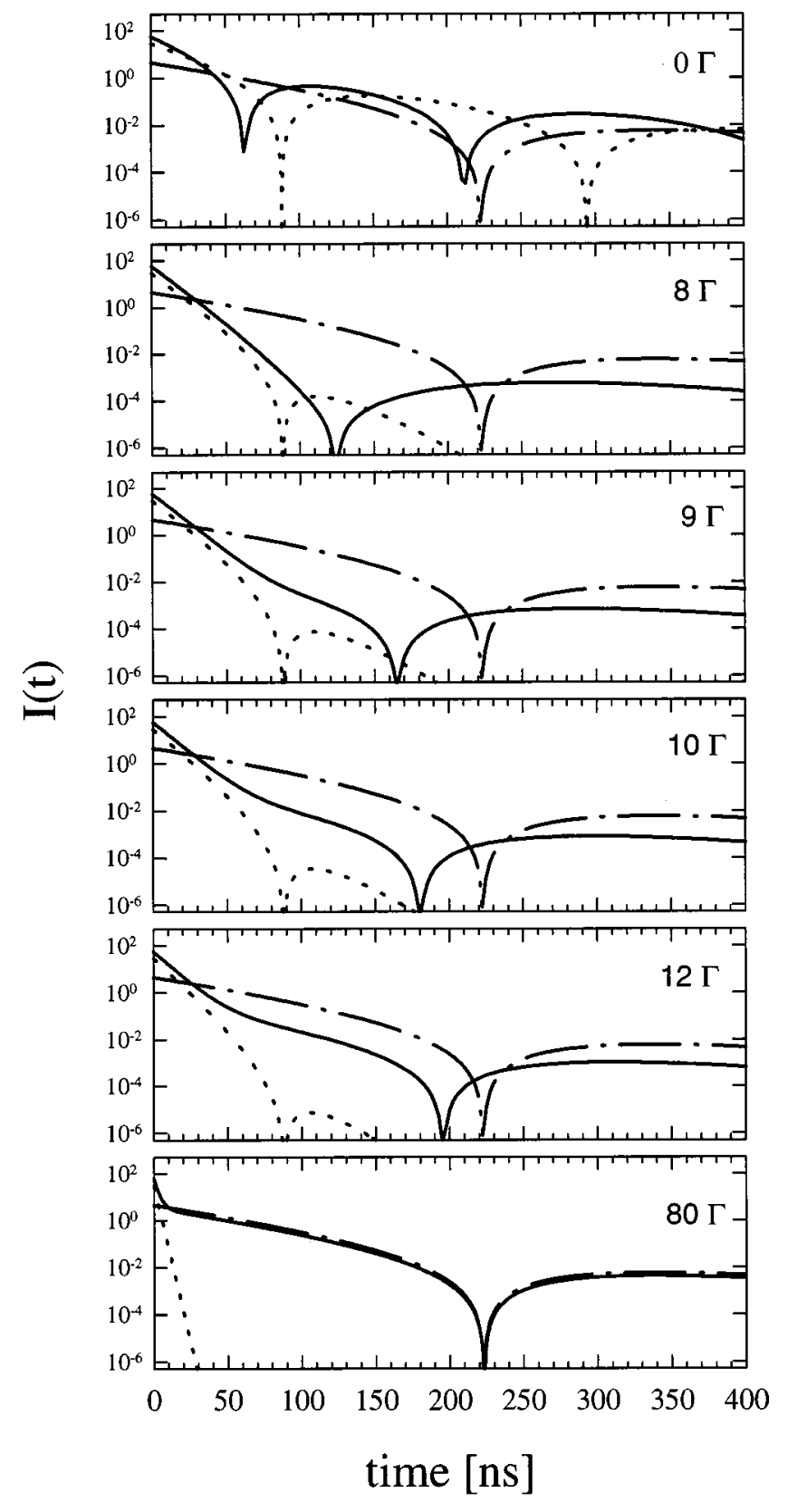

FIG. 7. Computer simulations of the NFSSR for target $A$ alone (dashed lines), target $B$ alone (dash-dotted lines), and the combined $(A+B)$ target (solid lines) for values of the additional Lorentzian broadening $\Delta_{A}$ for target $A$ in the range from $0 \Gamma$ to $80 \Gamma$. Note that for large $\Delta_{A}$ values the combined target $(A+B)$ exhibits a less steep initial $(t \approx 0)$ slope than for target $A$ alone (compare also Fig. 8 below). At later times the NFSSR of $(A+B)$ resembles that of target $B$ alone, although at a reduced intensity. Target $A, T_{A}$ $\approx 23.6$; $\operatorname{target} B, T_{B} \approx 9.3$.

coupling which drastically decreases the nuclear excitation amplitude in $\operatorname{target} B$ and thus reduces the radiation field reemitted by $\operatorname{target} B$. It would emit much more strongly at later times, if it were not driven by target $A$.

We now look at the results of simulations for the time dependences of the NFSSR intensity. Figure 7 shows simulations that cover the range of additional Lorentzian broad- 
ening of target $A$ up to $\Delta_{A}=80 \Gamma$. Both targets are kept stationary. $A B$ coupling is strongest if no additional broadening is present. Then simply a thicker target is formed, which exhibits higher initial intensity, enhanced decay rate, and earlier DB minima as compared to the corresponding properties of the individual targets (see Fig. 7, top). Since there is no dephasing a strong coherent signal from $\operatorname{target} A$ is visible until late times. The same holds for target $B$ and for the combined target $(A+B)$ where the intensity of the latter, in general, is larger than that of each individual target alone. When $\Delta_{A}$ is increased $A B$ coupling is reduced because of reduced overlap of the two sample resonances on the energy scale. This decrease in $A B$ coupling is indicated in Fig. 7 by the shift of the DB minima to later times $[16,22]$.

In the intermediate region $\left(8 \Gamma \leqslant \Delta_{A} \leqslant 12 \Gamma\right)$ both $A B$ coupling and dephasing determine the time evolution. Here at later times the combined targets emit much less intensity than $\operatorname{target} B$ alone would do. For example, at $\Delta_{A} \leqslant 12 \Gamma$, the intensity emitted by $(A+B)$ at late times $(t \geqslant 300 \mathrm{~ns})$ is about a factor of 10 less than that of $B$ alone. In particular, in the time window between 60 and $160 \mathrm{~ns}$, this reduction and the simultaneous occurrence of the DB minimum cause reduction of the NFSSR intensity from the combined target by a factor of 10-100 with respect to the response of target $B$ alone.

For very large broadenings $\left(\Delta_{A}=80 \Gamma\right)$, the $A B$ coupling is so weak, except for very early times $(t \leqslant 20 \mathrm{~ns})$, that the two foils scatter almost independently. As will be shown below [see Sec. IIIC 2, Eqs. (8) and (9)], at $t \rightarrow 0$ the $A B$ coupling is independent of $q_{A}$ and $q_{B}$ and therefore at $t \rightarrow 0$ it is not diminished by inhomogeneous broadening; only at later times does this occur. If $\Delta_{A}$ is made very large $\left(\Delta_{A}\right.$ $=80 \Gamma$, Fig. 7) $A B$ coupling can be effective only at and from very early times [see Eq. (7)], where the signal from target $A$ is still strong, in spite of the rapid dephasing of the oscillators in $A$. After this dephasing the NFSSR pattern of the combined target is very similar to that of $\operatorname{target} B$ alone although at a slightly reduced level of intensity (see Fig. 7). The amount of reduction is determined by the coupling term in Eq. (7), which depends on $\Delta_{A}$.

\section{Initial slope of the time dependences}

Figure 7 shows that for large values of $\Delta_{A}$ the initial slope for $(A+B)$ is less steep than for $A$ alone. This is exhibited in greater detail in the computer simulations of Fig. 8, which shows the time dependences from $t=0$ up to $50 \mathrm{~ns}$. It is seen that for small values of $\Delta_{A}$ the initial slope for the combined target $(A+B)$ is steeper than that for target $A$ alone, whereas for values of $\Delta_{A} \geqslant 12 \Gamma$ this relation is inverted. This behavior can be understood from Eq. (7).

Using Eq. (7) for the reemitted radiation, the field at $t$ $=0$ is determined by $E(t) \propto\left(T_{A}+T_{B}\right)$. Thus the initial intensity, being proportional to $\left(T_{A}+T_{B}\right)^{2}$, is enhanced; it is, however, independent of $q_{A}$ and $q_{B}$ [compare Eq. (5) for $t$ $=0]$.

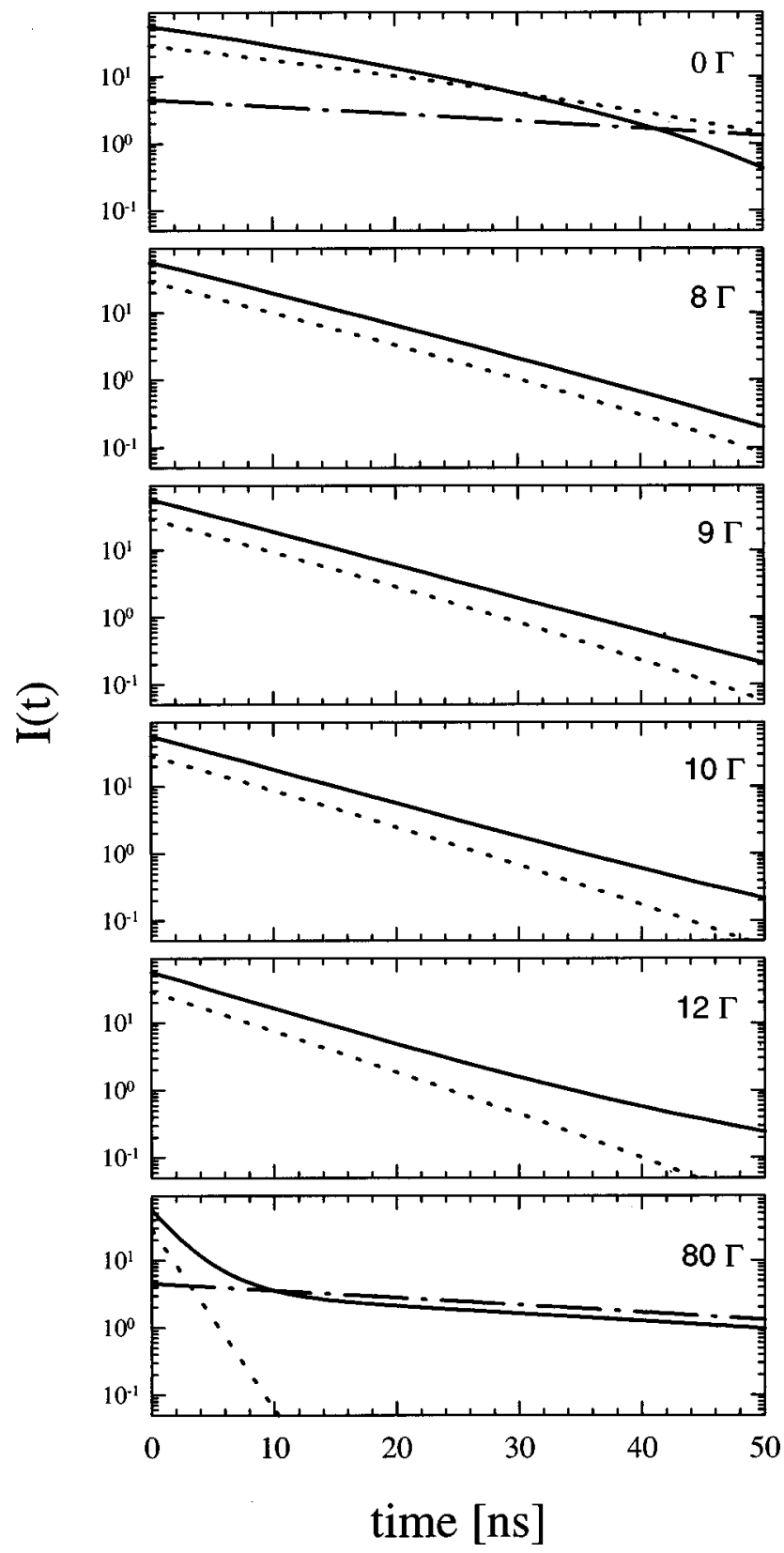

FIG. 8. Enlarged view of the NFSSR patterns of Fig. 7 at early times (between 0 and $50 \mathrm{~ns}$ ) showing the initial slopes of the time dependences. Other details as in Fig. 7.

The initial $(t \rightarrow 0)$ slopes of the intensity $d I_{A+B}(t) /\left.d t\right|_{t=0}$ and $d \ln I_{A+B}(t) /\left.d t\right|_{t=0}$ can be calculated as

$$
\begin{aligned}
d I_{A+B} /\left.d t\right|_{t=0} \propto & -(1 / 2 \tau)^{3}(1 / 2)\left[T_{A}^{2}\left(q_{A}+T_{A} / 4\right)\right. \\
& +T_{B}^{2}\left(q_{B}+T_{B} / 4\right)+T_{A} T_{B}\left(q_{A}+T_{A} / 4\right) \\
& \left.+T_{A} T_{B}\left(q_{B}+T_{B} / 4\right)+T_{A}^{2} T_{B} / 2+T_{A} T_{B}^{2} / 2\right]
\end{aligned}
$$

and 


$$
\begin{aligned}
d \ln I_{A+B} /\left.d t\right|_{t=0} \propto & -(1 / \tau) \frac{1}{\left(T_{A}+T_{B}\right)^{2}}\left[T_{A}^{2}\left(q_{A}+T_{A} / 4\right)\right. \\
& +T_{B}^{2}\left(q_{B}+T_{B} / 4\right)+T_{A} T_{B}\left(q_{A}+T_{A} / 4\right) \\
& \left.+T_{A} T_{B}\left(q_{B}+T_{B} / 4\right)+T_{A}^{2} T_{B} / 2+T_{A} T_{B}^{2} / 2\right] .
\end{aligned}
$$

Equations (8) and (9) show that there exist three different contributions to the initial slope.

(1) The first two terms in the square brackets describe the individual contributions of $\operatorname{target} A$ and $\operatorname{target} B$. These terms originate from the inhomogeneous line broadening characteristic for each target and from the coupling of the oscillators via the radiation field within each target. The coupling and inhomogeneous line broadening are represented by the Mössbauer thickness $T_{A, B}$ and by the additional Lorentzian broadening $q_{A, B}$, respectively.

(2) The next two terms in the square brackets of Eqs. (8) and (9) originate from the interference of the two wave fields reemitted individually from target $A$ and from target $B$. These two terms also depend on $T_{A, B}$ and $q_{A, B}$.

(3) The last two terms in the square brackets of Eqs. (8) and (9) originate from the integral term of Eq. (7) and describe the $A B$ coupling by the radiation field from $A$ impinging onto $B$. These terms depend on $T_{A, B}$; however, they are independent of $q_{A, B}$ (for $t \rightarrow 0$ ). It may seem surprising that for $t \rightarrow 0$ the $A B$ coupling is independent of the additional broadening, whereas the other two contributions do depend on $q_{A, B}$. This can be understood qualitatively: coupling within a resonance target is determined only by the effective thickness of the target and in the case of Lorentzian broadening does not depend on the resonance width (see, e.g., $[37,41]$ and references therein). An increase of the width causes an additional increase of the NFSSR decay rate because of dephasing. As to the $A B$ coupling, it is sensitive to the resonance width only if there exists a difference of the widths of the resonances in the targets $A$ and $B$ [see the integrand in Eq. (7)]. At the initial stage of the decay where both resonances are strongly broadened according to the time-energy uncertainty principle $[13,22], q_{A, B}$ become unimportant. In such conditions the $A B$ coupling is maximal. At later stages of the decay the dependence on $q_{A, B}$ is restored and $A B$ coupling is reduced.

Equation (9) can also be written as

$$
\begin{array}{r}
d \ln I_{A+B} /\left.d t\right|_{t=0} \propto-(1 / \tau)\left[\left(q_{A} T_{A}+q_{B} T_{B}\right) /\right. \\
\left.\left(T_{A}+T_{B}\right)+\left(T_{A}+T_{B}\right) / 4\right] .
\end{array}
$$

Here the first term in the sum gives the weighted slope of the initial decay in the kinematical approximation. The second term describes the effect of the total coupling, including both the coupling within each target and the $A B$ coupling. Note again that for $t \rightarrow 0$ the total coupling does not depend on $q_{A}$ and $q_{B}$.

For target $A$ alone we get $\left[T_{B}=0\right.$ in Eq. (10) $]$

$$
d \ln I_{A} /\left.d t\right|_{t=0}=-(1 / \tau)\left(q_{A}+T_{A} / 4\right)
$$

The initial slopes defined by Eqs. (10) and (11) are equal if

$$
q_{A}=q_{B}+\left(T_{A}+T_{B}\right) / 4,
$$

i.e., if the Lorentzian broadening in $A$ is larger than $q_{B}$ by an amount that is determined by the total coupling.

If $q_{A}<q_{B}+\left(T_{A}+T_{B}\right) / 4$, the slope of $(A+B)$ is steeper, whereas if $q_{A}>q_{B}+\left(T_{A}+T_{B}\right) / 4$, the slope of $A$ alone is steeper. In the present experiment, $q_{B} \approx 1.5$ and $\left(T_{A}+T_{B}\right)$ $\approx 33$ lead to equal initial slopes for $q_{A} \approx 10$, which corresponds to $\Delta_{A} \approx 9 \Gamma$ (compare Fig. 8).

According to Eq. (9), on the logarithmic scale, $d \ln I_{A+B} /\left.d t\right|_{t=0}$ is normalized to the intensity at $t=0$ by dividing by $\left(T_{A}+T_{B}\right)^{2}$, i.e., an average is taken over both targets. On a linear scale, however, where such a normalization is not performed, the slope for the combined target $d I_{A+B} /\left.d t\right|_{t=0}$ is always more negative than the slope $d I_{A} /\left.d t\right|_{t=0}$ for target $A$ alone.

\section{Dependence of $A B$ coupling on the relative shift of the resonance energy}

The $A B$ coupling can also be influenced by changing the resonance energy of the nuclei in one ensemble $[22,38,42,43]$. This can be achieved by Doppler-shifting one target $(B)$ with respect to the other $(A)$. Figure 9 displays a set of NFSSR time dependences recorded with target $A$ (at $20 \mathrm{~K}$ ) kept stationary and target $B$ (at room temperature) shifted by various Doppler energies $\Delta E$. Figure 10 shows a similar set of data obtained with target $A$ at $2.8 \mathrm{~K}$ and target $B$ again at room temperature and Doppler shifted.

With target $A$ at $20 \mathrm{~K}$, Fig. 9 exhibits the transition from pure dynamical beats at $\Delta E=0$ [Fig. 9(a)] via hybrid beats [44] (when $\Delta E$ is not larger than a few $\Gamma$ ) to quantum beats (QB's) for large $\Delta E[\Delta E \approx 87 \Gamma$, Fig. 9(f)]. For small values of $\Delta E$ up to a few $\Gamma$ [Figs. $9(\mathrm{a})-9(\mathrm{~d})]$ the coupling term is important. The beat patterns closely resemble those of a dynamical beat except that with increasing $\Delta E$ the minima are shifted to earlier times. We want to emphasize that in contrast to the case of line broadening (see Fig. 7) now in the case of line separation the minima are shifted to earlier times. This effect is caused by destructive interference due to the phase evolution of the radiation fields emitted by oscillators of two slightly different energies [see the phase factor $\exp \left\{i\left(\omega_{A}-\omega_{B}\right) t\right\}$ in the second term of Eq. (7)]. This transition region of small values for $\Delta E$ has been described in greater detail in Refs. [18,22]. With $\Delta E \approx 87 \Gamma$, the two targets are essentially fully decoupled. This can also be seen from Eq. (7). Since $\left|\omega_{A}-\omega_{B}\right|$ is large, the integrand in the coupling term is a rapidly oscillating function which under integration causes this term to nearly vanish. QB's are formed due to the interference of the radiation fields emitted from $A$ and $B$ separately. At $t \approx 85 \mathrm{~ns}$ [see Fig. 9(f)] the amplitude (contrast) of the QB's is almost zero. After the minimum of contrast the phase of the QB is shifted by $\pi$. This is due to the passage through zero of $A^{u}(t)$ for the thicker target (compare the dotted line in Fig. 6) [17,22]. This is another indication of complete decoupling. 


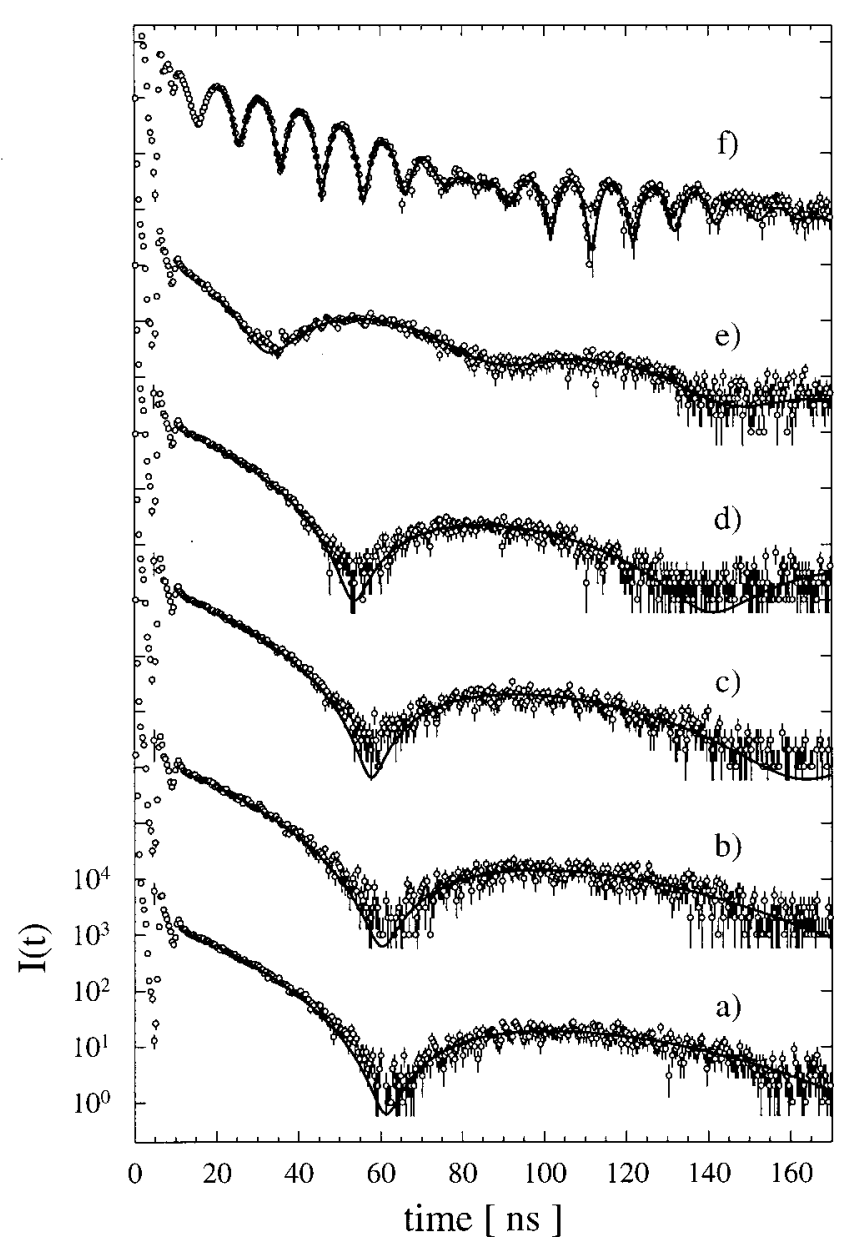

FIG. 9. NFSSR of the combined target $(A+B)$ with target $A$ (at $20 \mathrm{~K}$ ) kept stationary and target $B$ (at room temperature) Doppler shifted by $0.03 \Gamma$ (a), $1.3 \Gamma$ (b), $2.5 \Gamma$ (c), 4.0 (d), $11 \Gamma(\mathrm{e})$, and $87 \Gamma$ (f) relative to target $A$. For other details see Figs. 3 and 4.

Figure 10 shows the results when target $A$ is cooled to 2.8 $\mathrm{K}$ and exhibits substantial line broadening corresponding to $\Delta_{A} \approx 12 \Gamma$. Figure 10 (a) depicts the time dependence when $\Delta E$ is still kept small $(\Delta E \approx 0.3 \Gamma)$ by moving target $B$ to compensate for the SOD shift. The nuclear oscillators of both targets contribute constructively in the forward direction and, in addition, the $A B$ coupling, although reduced by the line broadening of target $A$, is still significant. However, in contrast to Fig. 9(a), the strong line broadening now leads to a completely different time evolution, as already discussed in Sec. III C in connection with Fig. 5(f). An enlarged decay rate (steeper slope) is observed at early times and the intensity is very low for times $t \geqslant 70 \mathrm{~ns}$, where target $B$ alone would still emit strongly.

In Fig. 10(b) target $B$ was kept stationary. The value $\Delta E \approx 1.2 \Gamma$ obtained from the fit is due to the second-order Doppler shift of the stainless-steel alloy between room temperature and $2.8 \mathrm{~K}$. An increased intensity between 60 and 110 ns compared to Fig. 10(a) is clearly discernible. This indicates a slight reduction of the $A B$ coupling that is responsible for the low intensity for times $t \geqslant 70 \mathrm{~ns}$ in Fig. 10(a).

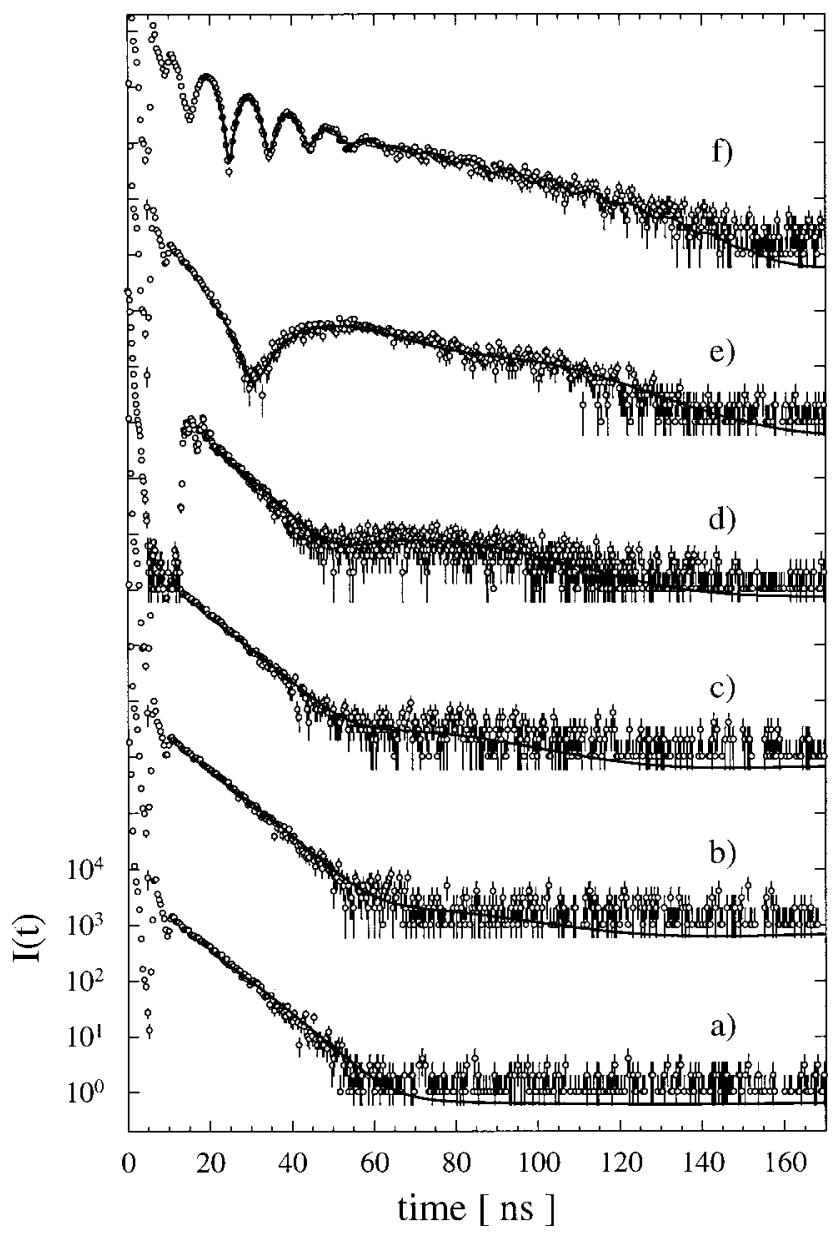

FIG. 10. NFSSR of the combined target $(A+B)$ with target $A$ (at $2.8 \mathrm{~K}$ ) kept stationary and $\operatorname{target} B$ (at room temperature) Doppler shifted by $0.3 \Gamma$ (a), $1.2 \Gamma$ (b), $2.1 \Gamma$ (c), 4.0 (d), $11 \Gamma$ (e), and $90 \Gamma$ (f) relative to target $A$. Curve (b) corresponds to the SOD shift of stainless steel between room temperature and $2.8 \mathrm{~K}$. For other details see Figs. 3 and 4.

When $\Delta E$ is increased further [see Figs. 10(c)-10(e)] the $A B$ coupling is also diminished further and a $\mathrm{QB}$ develops due to the interference of the radiation fields emitted from the two targets separately. The minimum in the time dependence at $t \approx 25 \mathrm{~ns}$ in Fig. 10(e) is already mainly caused by the QB's originating from the Doppler shift $\Delta E=11 \Gamma$. At $\Delta E=90 \Gamma$, target $A$ is essentially fully decoupled from target $B$ [see Fig. 10(f) and Eq. (7)]. A QB exhibiting a period of $\sim 10 \mathrm{~ns}$ corresponding to $\Delta E=90 \Gamma$ is visible as long as the signal from $A$ is still intense. The QB interference pattern almost vanishes for $t \geqslant 70 \mathrm{~ns}$ where the radiation field from $A$ alone [see Fig. 4(f)] has also been found to disappear. Since the two targets are essentially fully decoupled and target $A$ no longer radiates, the NFS pattern at later times ( $t \geqslant 70 \mathrm{~ns})$ is very similar to that of $\operatorname{target} B$ alone [compare Fig. 3(b)].

Thus the Doppler shift is a very effective method of destroying the $A B$ coupling. On the other hand, the time dependences react very sensitively even to small changes of the coupling and allow, for example, the determination of the SOD shift to a high precision. 


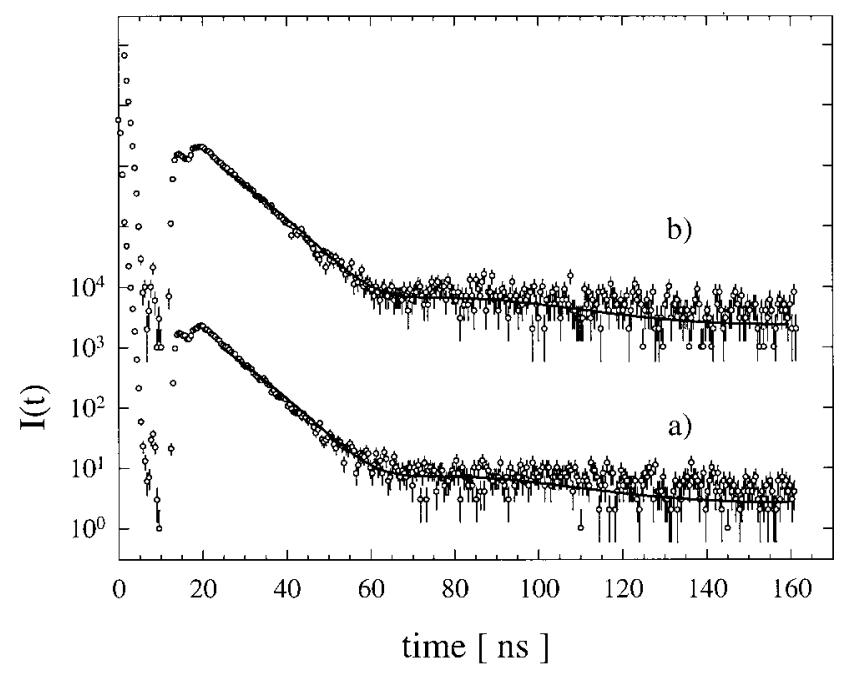

FIG. 11. Change of sequence of the targets. Target $B$ (at room temperature) downstream (a) and upstream (b) of target $A$ (at 2.8 $\mathrm{K})$. The targets were kept stationary. A SOD shift of $\sim 1.2 \Gamma$ [see Fig. 10(b)] is taken into account in the fits. Within statistical accuracy both NFSSR time dependences are identical.

\section{E. Sequence of targets}

Since target $A$ has different values for thickness and linewidth from target $B$ the experiment might not be symmetric with respect to an interchange of the targets, i.e., the NFSSR through the two targets might not be independent of the target sequence. Nevertheless, on the basis of Eq. (7) the invariance of NFSSR can be shown [22,39]. Clearly, if no coupling term were present, Eq. (7) would be symmetric, i.e., it would remain the same under interchange of $A$ and $B$. However, it is also symmetric if the integral term is included: Interchanging $A$ and $B$ in the integral term results in a mutual replacement of the time variables $t^{\prime} \Leftrightarrow t-t^{\prime}$, which corresponds to a shift in time. Taking into account that $-\int_{t}^{0} d t^{\prime}$ $=\int_{0}^{t} d t^{\prime}$ it is seen that Eq. (7) is invariant under interchange of $A$ and $B$.

This invariance can also be shown directly in energy space: in nuclear forward scattering, the response function is given by the transmission amplitude $[R(\omega)$ in Ref. [20]]. The response function for the combined target is given by the product of the transmission amplitudes of the constituent targets which is invariant to the order of the individual factors.

In Fig. 11, two measured time dependences are compared, with target $B$ downstream of target $A$ [Fig. 11(a)] and vice versa [Fig. 11(b)]. Within the statistical accuracy there is no difference between the two patterns. Thus it is also experimentally confirmed that the target sequence is of no importance in the case analyzed.

Still it is interesting to gain additional physical insight. As mentioned in Sec. IIIC 1 above, one should combine the second and the integral terms in Eq. (7) to get the total response of $\operatorname{target} B$. In this notion the radiation field reemitted by $\operatorname{target} B$ reflects both that $\operatorname{target} B$ is charged by the SR pulse and that it is driven by the radiation field reemitted by target $A$.

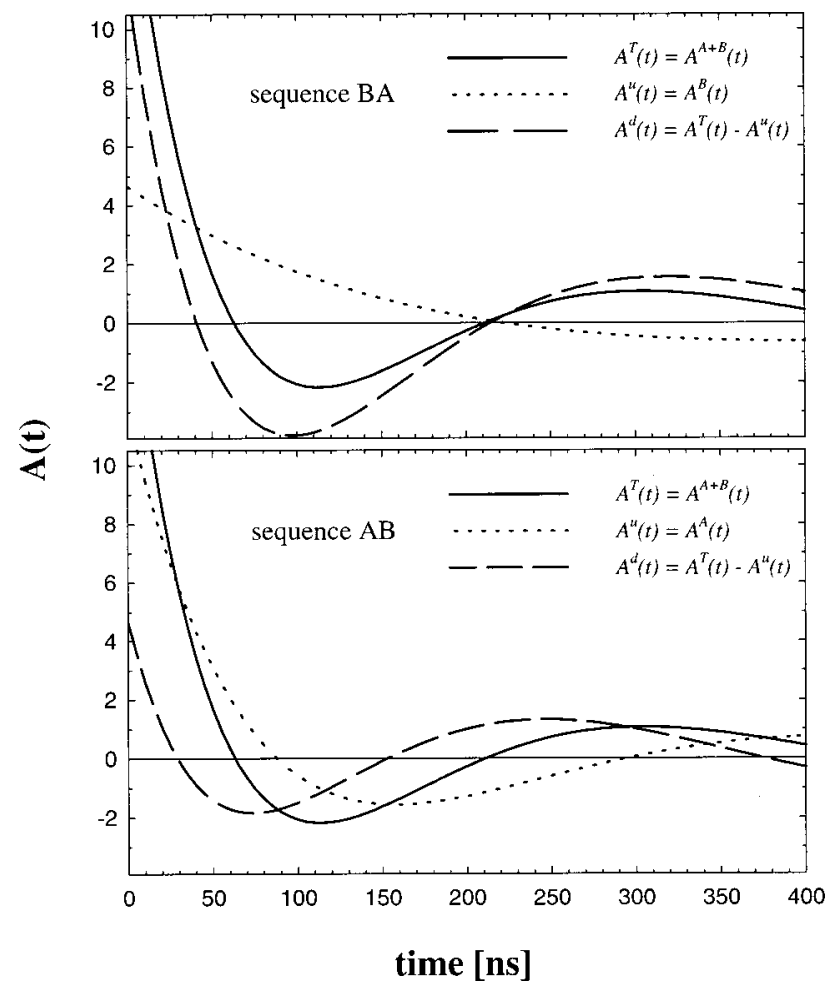

FIG. 12. Envelopes of the radiation fields according to Eq. (2) of the combined $(A+B)$ target $A^{T}(t)$, of the upstream target $A^{u}(t)$, and of the downstream target $A^{d}(t)$ for the cases that $A$ is upstream of $B$ (bottom) and $B$ is upstream of $A$ (top). Other details for targets $A$ and $B$ as in Fig. 6.

Considering at first again the simple case (see Sec. III C 1) that both targets are stationary and kept at room temperature, Fig. 12 shows the envelopes $A(t)$ of Eq. (2) for the situations that $A$ is upstream of $B$ (bottom) and vice versa (top) [40]. Figure 12 (bottom) is the same as Fig. 6 and has been reproduced for direct comparison. The time evolution of the total amplitude $A^{T}(t)$ is identical in both panels of Fig. 12. However, looking at the same target $(A$ or $B)$, Fig. 12 clearly demonstrates that $A^{d}(t)$ when the target is in the downstream position, is changed quite strongly as compared to $A^{u}(t)$ when the same target is in the upstream position (compare, for instance, the zero crossings). Thus, if the radiation fields of $A$ and $B$ were measured separately, the target sequence would indeed matter. However, if both targets remain together the radiation intensity measured by the detector is independent of the target sequence in spite of the striking difference of the radiation fields of $A$ and $B$ between the two sequences.

Even more interesting is the case when target $A$ is kept at reduced temperature and exhibits additional line broadening. As discussed in Sec. III C 2, if $A$ is upstream of $B$, the radiation field of $A$ will rapidly decrease due to dephasing, and, because of $A B$ coupling, target $B$ will also emit only weak radiation at late times.

If foil $B$ is upstream of foil $A$ the situation will be quite different. The radiation field of $B$ being emitted from an oscillator system having unbroadened resonances exhibits the usual DB pattern, i.e., foil $B$ still emits strongly at later 
times also. The field of $A$ would be diminished rapidly due to dephasing if $A$ were present just by itself. However, due to $B A$ coupling the oscillators of $A$ are now driven in addition by the radiation field emitted by $B$. The field reemitted by target $A$ is then shifted by $\pi$, as discussed in Sec. III C 1 . Thus at late times each target still emits strongly in the forward direction. However, the two radiation fields are opposite in phase, which leads to the drastically reduced intensity observed in the detector for this target sequence also. Further experiments allowing determination of these phase shifts and amplitudes and the consequences of the inversion of the target sequence are under way.

\section{CONCLUSIONS}

We have investigated the time response of two spatially separated ensembles of nuclear oscillators to excitation by a SR pulse. The two ensembles were provided by two stainless-steel targets $A$ and $B$ mounted downstream behind each other. Cooling one ensemble $(A)$ to cryogenic temperatures made it possible to greatly increase the linewidth of this system by inhomogeneous broadening while system $B$ being kept at room temperature exhibited almost natural linewidth.

Inhomogeneous broadening causes rapid dephasing of the nuclear oscillators. The radiative coupling between the rapidly dephasing ensemble and the regular oscillator system of almost natural linewidth was investigated. In the target sequence $A B$, a strong influence of the dephasing ensemble $(A)$ on the regular system $(B)$ was observed. This $A B$ coupling causes $\operatorname{target} B$ to radiate at a high decay rate (steep slope) at early times and to emit only little intensity at late times of the NFSSR pattern. The $A B$ coupling decreases with increasing inhomogeneous broadening and can be destroyed very effectively by changing the resonance frequency of the nuclear ensemble of one target. This was shown experimentally by Doppler-shifting one target with respect to the other by means of a constant-velocity drive.

At early times after the SR pulse the decay rate of the NFSSR intensity for the combined target $(A+B)$ is usually much larger than that for each individual target alone. Radiative coupling within and between the two targets and inhomogeneous broadening are the major contributions to this enlarged decay rate. However, on a logarithmic scale the initial slope of the intensity for the combined target $(A$ $+B)$ can be less steep than for one target $(A)$ alone if the inhomogeneous broadening of this target is sufficiently large.

In all experiments performed in this investigation, the sequence of the targets does not matter. This has also been shown theoretically. However, the radiation fields emitted by each individual target can be very different and can very well depend on the target sequence. From this point of view, the observed independence of NFSSR of the target sequence is a striking feature. This question will be the focus of future investigations.

\section{ACKNOWLEDGMENT}

This work was supported by the Bundesministerium für Bildung, Wissenschaft, Forschung und Technologie under Contract Nos. 05 643WOA/SK8WOA and 05 643GUA1.
[1] J. B. Marion, Classical Dynamics of Particles and Systems, 2nd ed. (Academic, New York, 1970), p. 408.

[2] P. Pleshko and I. Palo'cz, Phys. Rev. Lett. 22, 1201 (1969).

[3] G. L. Lamb, Jr., Rev. Mod. Phys. 43, 99 (1971).

[4] L. Allen and J. H. Eberly, Optical Resonance and Two-Level Atoms (Wiley, New York, 1975).

[5] A. D. Linde, Inflation and Quantum Cosmology (Academic, New York, 1990).

[6] A. D. Linde, D. A. Linde, and A. Mezhlumian, Phys. Rev. D 49, 1783 (1994).

[7] F. J. Lynch, R. E. Holland, and M. Hamermesh, Phys. Rev. 120, 513 (1960).

[8] E. Ikonen, P. Helistö, T. Katila, and K. Riski, Phys. Rev. A 32, 2298 (1985).

[9] I. Tittonen, M. Lippmaa, P. Helistö, and T. Katila, Phys. Rev. B 47, 7840 (1993).

[10] Yu. V. Shvyd'ko, S. L. Popov, and G. V. Smirnov, J. Phys.: Condens. Matter 5, 1557 (1993); 5, 7047(E) (1993).

[11] Yu. V. Shvyd'ko, G. V. Smirnov, S. L. Popov, and T. Hertrich, Pis'ma Zh. Eksp. Teor. Fiz. 53, 69 (1991) [JETP Lett. 53, 69 (1991)].

[12] E. Gerdau and U. van Bürck, in Resonant Anomalous X-Ray Scattering, edited by G. Materlik, C. J. Sparks, and K. Fischer (North-Holland, Amsterdam, 1994), p. 589.

[13] G. V. Smirnov, Hyperfine Interact. 97/98, 551 (1996); in
X-Ray and Inner-Shell Processes, edited by R. L. Johnson, H. Schmidt-Böcking, and B. F. Sonntag, AIP Conf. Proc. No. 389 (AIP, Woodbury, NY, 1997), p. 323.

[14] Nuclear Resonant Scattering of Synchrotron Radiation, edited by E. Gerdau and H. de Waard; Hyperfine Interact. 123/124 (1999); 125 (2000).

[15] Yu. V. Shvyd'ko, Phys. Rev. B 59, 9132 (1999). The program was implemented in the code MOTIF by Yu. V. Shvyd'ko.

[16] W. Potzel, in Condensed Matter Studies by Nuclear Methods, Proceedings of the XXXIV Zakopane School of Physics, edited by E. A. Görlich and A. T. Pedziwiatr (Wydawnictwo Uniwersytetu Jagiellonskiego, Krakow, 1999), p. 95.

[17] U. van Bürck, Hyperfine Interact. 123/124, 483 (1999).

[18] U. van Bürck, W. Potzel, P. Schindelmann, Yu. V. Shvyd'ko, E. Gerdau, O. Leupold, and H. D. Rüter, Phys. Rev. A 61, 013803 (2000).

[19] J. B. Hastings, D. P. Siddons, U. van Bürck, R. Hollatz, and U. Bergmann, Phys. Rev. Lett. 66, 770 (1991).

[20] Yu. Kagan, A. M. Afanas'ev, and V. G. Kohn, J. Phys. C 12, 615 (1979).

[21] J. P. Hannon and G. T. Trammell, in Resonant Anomalous X-Ray Scattering (Ref. [12]), p. 565.

[22] G. V. Smirnov, Hyperfine Interact. 123/124, 31 (1999).

[23] G. V. Smirnov, Zh. Eksp. Teor. Fiz. (to be published) [JETP (to be published)]. 
[24] D. C. Burnham and R. Y. Chiao, Phys. Rev. 188, 667 (1969).

[25] Yu. Kagan, Hyperfine Interact. 123/124, 83 (1999).

[26] J. P. Hannon and G. T. Trammell, Hyperfine Interact. 123/124, 127 (1999).

[27] G. V. Smirnov, U. van Bürck, J. Arthur, S. L. Popov, A. Q. R. Baron, A. I. Chumakov, S. L. Ruby, W. Potzel, and G. S. Brown, Phys. Rev. Lett. 77, 183 (1996).

[28] D. P. Siddons, J. B. Hastings, and G. Faigel, Nucl. Instrum. Methods Phys. Res. A 266, 329 (1988).

[29] The high-resolution monochromator which was used at HASYLAB (Hamburg) employs channel-cut $\mathrm{Si}(422)$ and $\mathrm{Si}(1222)$ crystals in the nested geometry. It was designed by E. Gerdau, R. Rüffer, and H. D. Rüter, based on a proposal by T. Ishikawa, Y. Yoda, K. Izumi, C. K. Suzuki, X. W. Zhang, M. Ando, and S. Kikuta, Rev. Sci. Instrum. 63, 1015 (1992). For further details, see O. Leupold, E. Gerdau, H. D. Rüter, W. Meyer-Klaucke, A. X. Trautwein, and H. Winkler, in HASYLAB Annual Report I, 1996 (unpublished), p. 73.

[30] A. Q. R. Baron, Nucl. Instrum. Methods Phys. Res. A 352, 665 (1995).

[31] A. Q. R. Baron, R. Rüffer, and J. Metge, Nucl. Instrum. Methods Phys. Res. A 400, 124 (1997).

[32] S. Kishimoto, J. Synchrotron Radiat. 5, 275 (1998).

[33] U. Gonser, C. J. Meechan, A. H. Muir, and H. Wiedersich, J. Appl. Phys. 34, 2373 (1963).
[34] K. Umeda, Y. Obata, T. Toriyama, T. Inamura, and H. Iijima, Hyperfine Interact. 69, 485 (1991).

[35] W. Potzel et al. (unpublished).

[36] U. van Bürck, D. P. Siddons, J. B. Hastings, U. Bergmann, and R. Hollatz, Phys. Rev. B 46, 6207 (1992).

[37] Yu. V. Shvyd'ko, U. van Bürck, W. Potzel, P. Schindelmann, E. Gerdau, O. Leupold, J. Metge, H. D. Rüter, and G. V. Smirnov, Phys. Rev. B 57, 3552 (1998).

[38] W. Potzel, U. van Bürck, P. Schindelmann, G. V. Smirnov, E. Gerdau, Yu. V. Shvyd'ko, and H. D. Rüter, in HASYLAB Annual Report I, 1998 (unpublished), p. 953.

[39] G. V. Smirnov and W. Potzel, Hyperfine Interact. 123/124, 633 (1999).

[40] P. Schindelmann, Ph.D. thesis Technische Universität München, D-85748 Garching, Germany (1999).

[41] G. V. Smirnov and V. G. Kohn, Phys. Rev. B 52, 3356 (1995).

[42] W. Potzel, U. van Bürck, P. Schindelmann, G. V. Smirnov, E. Gerdau, O. Leupold, H. D. Rüter, and S. Schwendy, in HASYLAB Annual Report I, 1996 (unpublished), p. 882.

[43] U. van Bürck, W. Potzel, P. Schindelmann, G. V. Smirnov, E. Gerdau, O. Leupold, Yu. V. Shvyd'ko, and H. D. Rüter, in HASYLAB Annual Report I, 1997 (unpublished), p. 939.

[44] Yu. V. Shvyd'ko and U. van Bürck, Hyperfine Interact. 123/ 124, 511 (1999). 